\title{
Development of an airborne molecular direct detection Doppler lidar for tropospheric wind profiling
}

Bruce Gentry $^{\mathrm{a}}$, Matthew McGill ${ }^{\mathrm{a}}$, Geary Schwemmer ${ }^{\mathrm{b}}$, Michael Hardesty ${ }^{\mathrm{c}}$, Thomas Wilkerson ${ }^{\mathrm{d}}$, Marcos Sirota ${ }^{\mathrm{f}}$, Scott Lindemann ${ }^{\mathrm{g}}$, Floyd Hovis ${ }^{\mathrm{h}}$

\begin{abstract}
Global measurement of tropospheric winds is a key measurement for understanding atmospheric dynamics and improving numerical weather prediction. Global wind profiles remain a high priority for the operational weather community and also for a variety of research applications including studies of the global hydrologic cycle and transport studies of aerosols and trace species. In addition to space based winds, high altitude airborne Doppler lidar systems flown on research aircraft, UAV's or other advanced sub-orbital platforms would be of great scientific benefit for studying mesoscale dynamics and storm systems such as hurricanes. The Tropospheric Wind Lidar Technology Experiment (TWiLiTE) is a three year program to advance the technology readiness level of the key technologies and subsystems of a molecular direct detection wind lidar system by validating them, at the system level, in an integrated airborne lidar system. The TWiLiTE Doppler lidar system is designed for autonomous operation on the WB57, a high altitude aircraft operated by NASA Johnson. The WB57 is capable of flying well above the midlatitude tropopause so the downward looking lidar will measure complete profiles of the horizontal wind field through the lower stratosphere and the entire troposphere. The completed system will have the capability to profile winds in clear air from the aircraft altitude of $18 \mathrm{~km}$ to the surface with $250 \mathrm{~m}$ vertical resolution and $<3 \mathrm{~m} / \mathrm{s}$ velocity accuracy. Progress in technology development and status of the instrument design will be presented.

a NASA Goddard Space Flight Center, Laboratory for Atmospheres, Code 613.1, Greenbelt, MD 20771

Email: Bruce.M.Gentry@nasa.gov ; Ph : 301-614-6271

${ }^{\mathrm{b}}$ Science Engineering Services Inc, Columbia MD

${ }^{c}$ NOAA OAR/ESRL, Boulder CO

${ }^{\mathrm{d}}$ Utah State University Space Dynamics Laboratory, Logan, UT

e NOAA AOML, Miami FL

${ }^{f}$ Sigma Space Corp, Lanham MD

${ }^{\mathrm{g}}$ Michigan Aerospace Corporation, Ann Arbor, MI

${ }^{\mathrm{h}}$ Fibertek, Inc, Herndon, VA
\end{abstract}




\title{
Development of an airborne molecular direct detection Doppler lidar for tropospheric wind profiling
}

\author{
Bruce Gentry $^{1}$, Matthew McGill ${ }^{1}$, Geary Schwemmer ${ }^{2}$, Michael Hardesty $^{3}$, Alan Brewer ${ }^{3}$, \\ Thomas Wilkerson $^{4}$, Robert Atlas ${ }^{5}$, Marcos Sirota ${ }^{6}$, Scott Lindemann ${ }^{7}$, Floyd Hovis ${ }^{8}$ \\ ${ }^{I}$ NASA Goddard Space Flight Center, Laboratory for Atmospheres, Code 613.1, Greenbelt, MD 20771 \\ ${ }^{2}$ Science Engineering Services Inc, Columbia MD \\ ${ }^{3}$ NOAA ESRL, Boulder CO \\ ${ }^{4}$ Utah State University Space Dynamics Laboratory, Logan, UT \\ ${ }^{5}$ NOAA AOML, Miami FL \\ ${ }^{6}$ Sigma Space Corp, Lanham MD \\ ${ }^{7}$ Michigan Aerospace Corporation, Ann Arbor, MI \\ ${ }^{8}$ Fibertek Inc, Herndon, VA
}

\begin{abstract}
Global measurement of tropospheric winds is a key measurement for understanding atmospheric dynamics and improving numerical weather prediction. Global wind profiles remain a high priority for the operational weather community and also for a variety of research applications including studies of the global hydrologic cycle and transport studies of aerosols and trace species. In addition to space based winds, high altitude airborne Doppler lidar systems flown on research aircraft, UAV's or other advanced sub-orbital platforms would be of great scientific benefit for studying mesoscale dynamics and storm systems such as hurricanes. The Tropospheric Wind Lidar Technology Experiment (TWiLiTE) is a three year program to advance the technology readiness level of the key technologies and subsystems of a molecular direct detection wind lidar system by validating them, at the system level, in an integrated airborne lidar system. The TWiLiTE Doppler lidar system is designed for autonomous operation on the WB57, a high altitude aircraft operated by NASA Johnson. The WB57 is capable of flying well above the mid-latitude tropopause so the downward looking lidar will measure complete profiles of the horizontal wind field through the lower stratosphere and the entire troposphere. The completed system will have the capability to profile winds in clear air from the aircraft altitude of $18 \mathrm{~km}$ to the surface with $250 \mathrm{~m}$ vertical resolution and $<3$ $\mathrm{m} / \mathrm{s}$ velocity accuracy. Progress in technology development and status of the instrument design will be presented.
\end{abstract}

\section{Introduction -}

The primary objective of the Tropospheric Wind Lidar Technology Experiment (TWiLiTE) project is to develop the key technologies and subsystems of a molecular direct detection Doppler wind lidar and integrate them into an airborne testbed to validate, at the system level, both the technologies and the measurement approach. To realize the full benefit of the demonstration, the target platform is the NASA WB57 high altitude aircraft. The WB57 flies at $18 \mathrm{~km}$ so that the nadir viewing lidar will be able to profile winds

\begin{tabular}{|l|c|}
\hline Parameter & \\
\hline $\begin{array}{l}\text { Velocity accuracy (LOS } \\
\text { projected) (m/s) }\end{array}$ & 2.0 \\
\hline Range of regard (km) & $0-18$ \\
\hline Vertical resolution (km) & 0.25 \\
\hline $\begin{array}{l}\text { Horizontal resolution (km) } \\
\text { (completion of 1 step stare scan } \\
\text { cycle) }\end{array}$ & 25 \\
\hline $\begin{array}{l}\text { Aircraft Groundspeed (m/s) } \\
\text { Nadir angle (deg) }\end{array}$ & 200 \\
\hline Scan pattern & 45 \\
\hline $\begin{array}{l}\text { Horizontal integration per LOS } \\
\text { (seconds)/ground track (km) }\end{array}$ & $10 / / 2$ \\
\hline
\end{tabular}

Table 1 - TWiLiTE measurement requirements 
through the full troposphere. This will provide a capability to simulate the downward viewing geometry of the spaceborne lidar and to investigate the effects of clouds, aerosols and other atmospheric phenomena on the measurement. The TWiLiTE project will establish an exit technology readiness level of TRL 5 for all key components and subsystems and will provide a critical milestone on the path to a future spaceborne tropospheric wind system. The TWiLiTE program

\begin{tabular}{|l|l|}
\hline & Typical \\
\hline Wavelength & $355 \mathrm{~nm}$ \\
\hline $\begin{array}{l}\text { HOE Telescope/Scanner } \\
\text { Aperture }\end{array}$ & $0.38 \mathrm{~m}$ \\
\hline Laser Linewidth (FWHH) & $<150 \mathrm{MHz} @ 355 \mathrm{~nm}$ \\
\hline Laser Energy/Pulse & $30 \mathrm{~mJ}$ \\
\hline Etalon FSR & $16.7 \mathrm{GHz}$ \\
\hline Etalon FWHH & $2.84 \mathrm{GHz}$ \\
\hline Etalon Peak Transmission & $>60 \%$ \\
\hline $\begin{array}{l}\text { Interference filter BW } \\
\text { (FWHH) }\end{array}$ & $220 \mathrm{pm}$ \\
\hline PMT Quantum Efficiency & $25 \%$ \\
\hline
\end{tabular}

Table 2-TWiLiTE Instrument parameters

objectives are consistent with the technology development roadmaps for both direct detection and hybrid (combined direct and coherent) Doppler lidar solutions. The TWiLiTE airborne direct detection molecular Doppler lidar system will be the first of its kind, and as such, can be flown as a stand alone instrument or with existing or future coherent Doppler lidars to explore the potential synergies of data products, systems and shared technologies of the hybrid approach.

The TWiLiTE project is a collaboration involving scientists and engineers from NASA Goddard Space Flight Center, NOAA CSD, Utah State University Space Dynamics Lab, Michigan Aerospace Corporation, Fibertek Inc and Sigma Space Corporation. The TWiLiTE instrument leverages significant research and development investments made by NASA Goddard and it's partners in the past several years in key lidar technologies and sub-systems (lasers, telescopes, scanning systems, detectors and receivers) required to enable spaceborne global wind lidar measurement

During the three year TWiLiTE program these subsystems will be integrated into a complete molecular direct detection Doppler wind lidar system designed for autonomous operation on a high altitude aircraft, such as the NASA WB57. The TWiLiTE instrument will be the first demonstration of an airborne scanning direct detection Doppler lidar and will serve as a critical milestone on the path to a future spaceborne tropospheric wind system. In addition to being a technology testbed for space based tropospheric wind lidar, when completed the TWiLITE high altitude airborne lidar will be used for studying mesoscale dynamics and storm research (e.g. winter storms, hurricanes) and can be used for calibration and validation of satellite based wind systems such as ESA's Aeolus Atmospheric Dynamics Mission (Stoffelen et al 2005). The TWiLiTE Doppler lidar will have the capability to profile winds in clear air from the aircraft altitude of $18 \mathrm{~km}$ to the surface with $250 \mathrm{~m}$ vertical resolution and $<2 \mathrm{~m} / \mathrm{s}$ velocity accuracy. Measurement requirements used in the design of TWiLiTE are listed in Table 1. The measurement requirements are used to define tracability from the system level to the individual subsystem and component technology requirements.

\section{TWiLiTE Design and Expected Performance -}

The TWiLiTE Doppler lidar is a molecular direct detection system operating at a wavelength of $355 \mathrm{~nm}$. The lidar system is composed of four major subsystems: 1) the single frequency pulsed laser transmitter; 2) The transceiver telescope and scanner; 3) The Doppler receiver and 4) the Command and Data Handling electronics that control the instrument functions and acquire and store the science data. System level integration of these components requires definition of mechanical, electrical, optical and thermal interfaces between the subsystems and from the system to the platform, in this case the WB57 aircraft. A common mechanical structure and electrical power distribution system is also being developed. A final requirement is the control interface. To be compatible with the WB57, which does not offer the option of flying an operator, the TWiLiTE Doppler lidar is designed to be fully autonomous in operation. There are only the top level commands to power the instrument on or off; allow or inhibit the laser firing and begin data acquisition. All other functions are controlled internally in flight.

To profile winds the laser transmits a pulse to the atmosphere and a fraction of the laser energy is backscattered from molecules and aerosols back towards the lidar. The backscattered signal is collected by the telescope and directed to the Doppler receiver which analyzes the signal to determine the frequency shift (Doppler shift) introduced by the mean velocity of the 
scatterers, e.g. the component of the horizontal wind velocity projected on the line of sight of the laser. In order to measure the horizontal component the laser and telescope are pointed off nadir at 45 degrees. A final requirement is the ability to point the lidar in azimuth in order to obtain radial winds from different directions. The multiple azimuth perspectives provide the necessary information to determine the vector wind field. Range, or height, resolved profiles are obtained by acquiring and processing the data for the entire time it takes the laser pulse to traverse the atmosphere from the aircraft to the surface. The time of flight of the laser provides precise determination of range.

TWiLiTE is a direct detection Doppler lidar designed to measure winds using the signal backscattered by molecules. The Doppler frequency shift is measured with a molecular double edge receiver implemented in a design that is similar to those described previously (Gentry et al 2000, Flesia and Korb 1999). The double edge method utilizes two high spectral resolution optical filters located symmetrically about the outgoing laser frequency to measure the Doppler shift. The double edge measurement concept is illustrated in Figure 1. A simulated atmospheric molecular and aerosol backscattered spectrum is shown for reference. To make the wind measurement, the two edge filter channels are located symmetrically about the outgoing laser frequency in the wings of the thermally broadened molecular Rayleigh-Brillouin molecular backscattered spectrum.

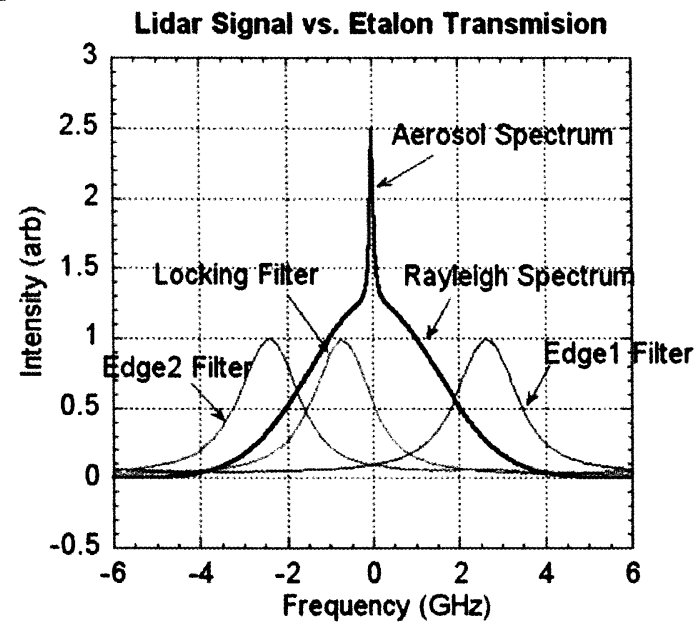

Figure 2 - Double Edge measurement concept. The two edge filter channels are shown symmetrically located about the outgoing laser frequency in the wings of the thermally broadened molecular backscattered spectrum.
The molecular system operates in the ultraviolet at 355 $\mathrm{nm}$ in order to take advantage of the $\lambda^{-4}$ dependence of the molecular scattering. Many of the design elements of the TWiLiTE lidar have been demonstrated and validated in ground-based lidar measurements (Gentry

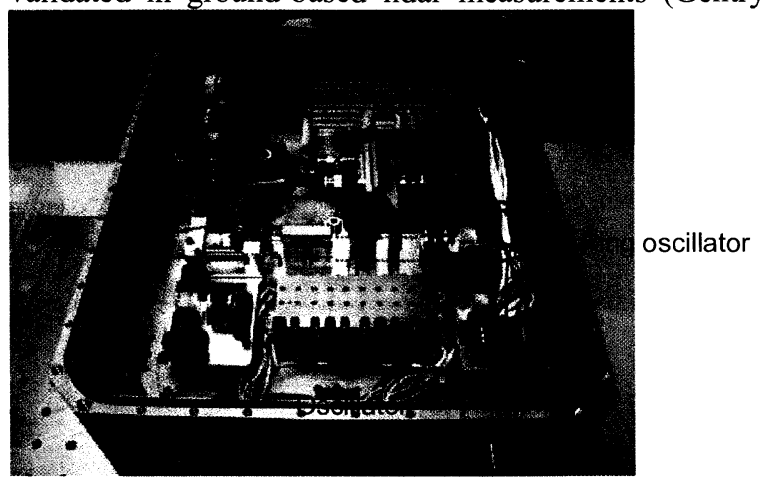

Figure 3 - Single frequency 355 nm Nd:YAGlaser

and Chen 2002). The TWiLiTE lidar system baseline performance characteristics are summarized in Table 2.

The transmitter is a single frequency, Nd:YAG laser frequency tripled to the third harmonic wavelength of $355 \mathrm{~nm}$. The laser pulse energy is nominally $30 \mathrm{~mJ}$ at $355 \mathrm{~nm}$ and the pulse repetition frequency is $200 \mathrm{~Hz}$. In order to obtain single frequency, narrowband output

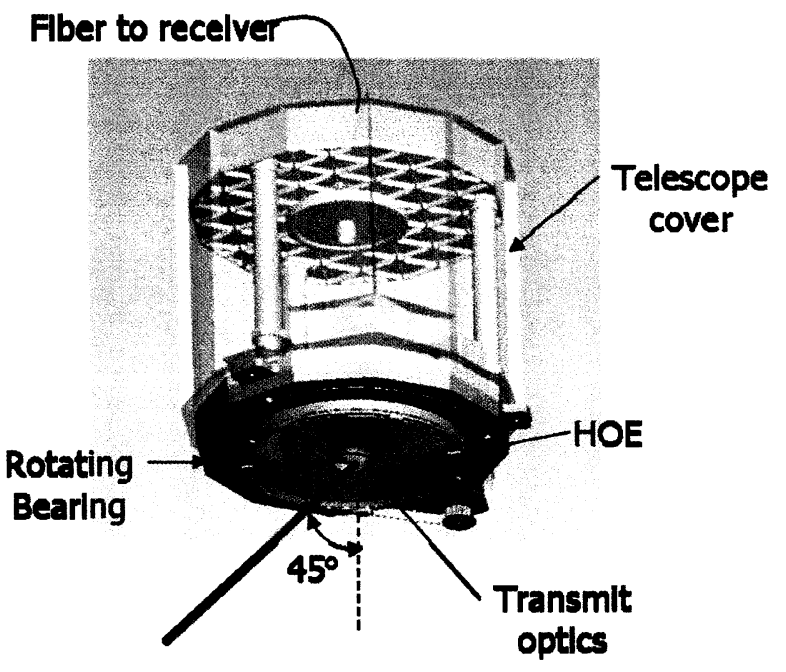

Figure 1 - Rotating HOE transceiver

pulses the laser is injection seeded using a ramp-and-fire resonance locking technique (Hovis et al 2003). The ramp and fire technique is particularly suitable for airborne operation as it is relatively insensitive to vibration. The laser is a master oscillator power amplifier (MOPA) configuration designed and built by Fibertek, Inc. The design has heritage to the High 
Brightness Laser, a NASA SBIR project that Fibertek completed in 2003. Since that time Fibertek has built several generations of this type of laser. Figure 2 shows a view of the internal layout of the laser. The pressurized canister, transparent in this view, has dimensions of $31 \mathrm{~cm} \times 25 \mathrm{~cm} \times 14 \mathrm{~cm}$. The laser draws about $480 \mathrm{~W}$ of electrical power from the aircraft $28 \mathrm{~V}$ supply.

The telescope and conical scanning functions will be accomplished with a $38 \mathrm{~cm}$ clear aperture, rotating holographic optical element (HOE) transceiver (Schwemmer et al 1998). The HOE transceiver subsystem performs both functions of transmitting the laser beam and receiving the atmospheric backscattered signal. The TWiLiTE transceiver, shown in Figure 3, contains a $40-\mathrm{cm}$ diameter rotating $\mathrm{HOE}$, laser beam steering and collimating optics, and a fiber optic interface to the Doppler receiver. The HOE aperture determines the receiver collecting area. The lidar measures the Doppler shift of the component of the wind projected on the line-of-sight of the laser beam. In order to resolve the horizontal wind the HOE telescope is designed to direct the beam at a nadir angle of $45^{\circ}$. Rotation of the HOE repeatedly sweeps the transmitted laser beam and the receiver's FOV through a $45^{\circ}$ cone about the axis of rotation. The scanner will step in azimuth to specified angles, typically 8 to 16 positions per scan cycle. After moving and settling to each fixed azimuth position the system will integrate signal for a period of 10 seconds (2000 laser shots). The backscattered signal collected by the HOE will be focused to a 200 micron core diameter multimode fiber optic which brings the collected signal to the Doppler receiver.
In the Doppler receiver, the collected signal is split into a total of four channels. Three of these beams are directed along parallel paths through a high spectral resolution tunable Fabry-Perot etalon which is used as the edge filter. As shown in Figure 4, the etalon has three sub-apertures corresponding to the filter bandpass functions labeled Edge1, Edge2 and Locking in Figure 1. The etalon channels have slightly different bandpass center frequencies but otherwise nearly identical optical properties e.g. peak transmission, finesse, free spectral range. The separation of the two edge filter center

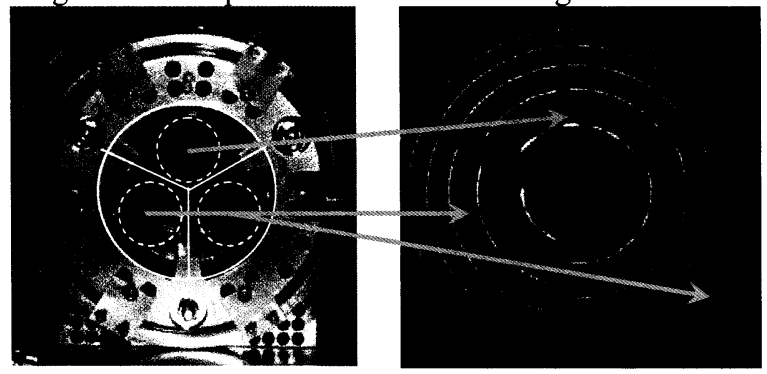

Figure 4 -The TWiLiTE Fabry Perot etalon (left) has three sub-apertures with bandpasses separated in frequency to produce the molecular double edge measurement arrangement described in the text. The three spectral regions are clearly seen in the full fring image of the Fabry Perot circular ring pattern shown on the right

wavelengths is chosen so the velocity sensitivity of the broader molecular signal, defined as the change in the ratio of the two edge channel transmittances for a Doppler shift of $1 \mathrm{~m} / \mathrm{s}$, is equal to the velocity sensitivity of the narrower aerosol signal. Matching the velocity sensitivities in this way greatly reduces the effects of aerosols on the wind measurements. The two etalon 'edge' channels have PMTs operating in photon

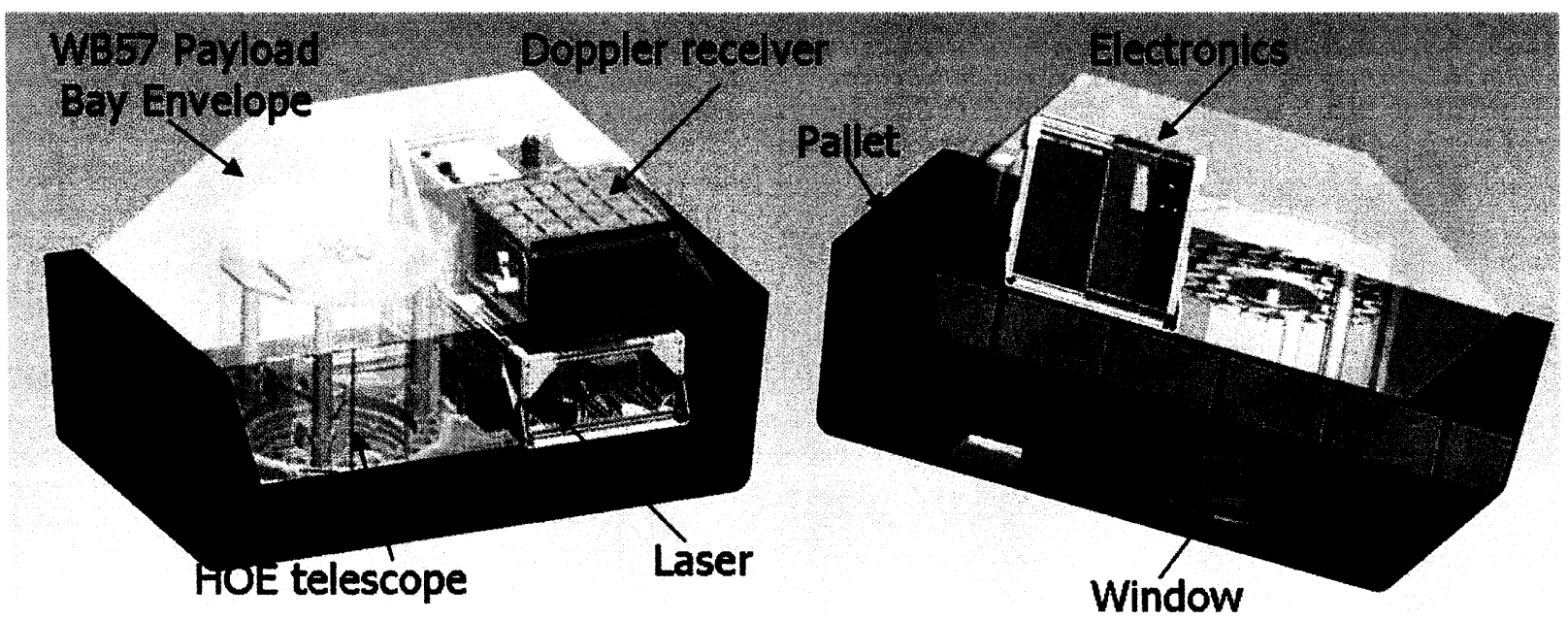

Figure 5 - Two views, side/top and side/bottom, of the TWiLiTE lidar system integrated on the WB57 three foot pallet 
counting mode. These channels provide the information used in the atmospheric Doppler shift measurement. The locking etalon peak is located such that the outgoing laser frequency is aligned to the half height point of the locking filter bandpass. This third etalon channel is used to sample the outgoing laser frequency and will be used as a reference in the Doppler shift measurement to correct for small frequency drifts of the laser or etalon. The fourth channel is an energy monitor used to provide intensity normalization of the respective etalon channels. In each of the detector channels there are three Hamamatsu photomultiplier tubes sharing the incoming signal to that channel in the intensity ratio of $90: 9: 1$.

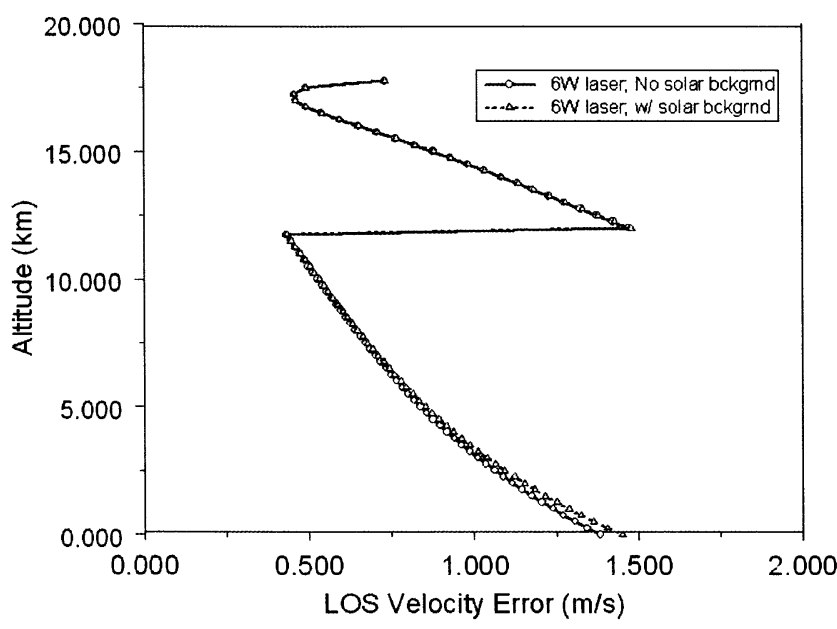

Figure 6 - Simulated line of sight velocity errors shown for a laser average power levels: $6 \mathrm{~W}(30 \mathrm{~mJ}, 200 \mathrm{pps})$. Errors are shown with and without solar background to represent day and night time performance.

This arrangement allows the signal dynamic range of the photon counting PMT's to be increased by two orders of magnitude. When the signal on the high signal detector $(90 \%)$ exceeds the maximum incident counting rate of 50 Mcts, the signal from that detector can be excluded from the wind analysis. The photon counting signals are binned in a multi-channel scalar, integrated for a selectable number of shots and stored.

These subsystems comprise the working elements of the TWiLiTE lidar system. They are integrated on a mechanical structure compatible with the WB57 research aircraft payload pallet (see Section 2.3). The laser and telescope are rigidly mounted to an optical bench to maintain bore sight and alignment. The instrument pallet also includes the laser electronics, command and data handling electronics, thermal management system and power distribution and control system. A view of the integrated TWiLiTE lidar system is shown in Figure 5. The estimated mass of the instrument is $515 \mathrm{lbs}$

The expected performance of the TWiLiTE lidar system has been simulated using detailed instrument models that fully describe the direct-detection Doppler lidar system (McGill et al 1999). The instrument models have been verified using the GLOW ground based molecular double edge Doppler lidar, and it is found that simulated performance matches actual system performance to 3$5 \%$ accuracy.(Gentry and Chen, 2002) Simulation of the TWiLiTE lidars performance have been performed using single profile aerosol model atmospheres. The simulated molecular and aerosol backscattered photon returns can be used along with a knowledge of the Doppler receiver characteristics to predict shot noise limited velocity accuracy. Solar background is also included to estimate expected daytime performance. The solar background is calculated using a worst case of a fully illuminated cloud (albedo values of 1.0) scene.

Figure 6 shows a simulation of expected line-of-sight wind velocity errors for the molecular Doppler lidar instrument flying on the WB57 aircraft flying at an altitude of $18 \mathrm{~km}$. As shown, line-of-sight wind errors are less than $1.5 \mathrm{~m} / \mathrm{s}$ from $18 \mathrm{~km}$ down to the surface for laser average power levels of $6 \mathrm{~W}$. In this simulation the vertical resolution is $250 \mathrm{~m}$ and 10 seconds of averaging is used. Note the discontinuity in the simulated error observed at about $12 \mathrm{~km}$ altitude. This is due to the signal on the high (90\%) PMT in the etalon Edge channels exceeding the threshold maximum count rate. For altitudes above that point only the signals from the medium (9\%) and low (1\%) PMTs are included. The simulated errors shown in Figure 6 include only shot noise although tracking of other error sources is being done and total random error form all source is not expected to exceed $2.0 \mathrm{~m} / \mathrm{s}$.

\subsection{TWiLiTE Development Status}

The TWiLITE IIP project officially started on August 2, 2005 and will end on August 1, 2008. In order to track the development progress we adopted a version of the review cycle used for flight projects at Goddard. A summary of the IIP project timeline is shown in Figure 7. The last of the major design reviews, the Critical Design Review, was completed on May 1, 2007. In the summer of 2007, at the completion of the second year, the laser, telescope, etalon and Doppler receiver are all scheduled to be completed and delivered. The third year of the IIP project will involve ground testing of the subsystems, completion of the command and data 
handling hardware and software and integration of all the modules into the complete instrument package, ready for integration to the WB57 instrument pallet. At that point in time we will be ready for the initial flight testing of the instrument.

\section{Summary}

The design features and key technologies being developed as part of the Tropopsheric Wind Lidar Technology Experiment (TWiLiTE) IIP project have been described. The TWiLiTE airborne lidar will present several new and challenging problems. A major

TWLITE Project Timeline

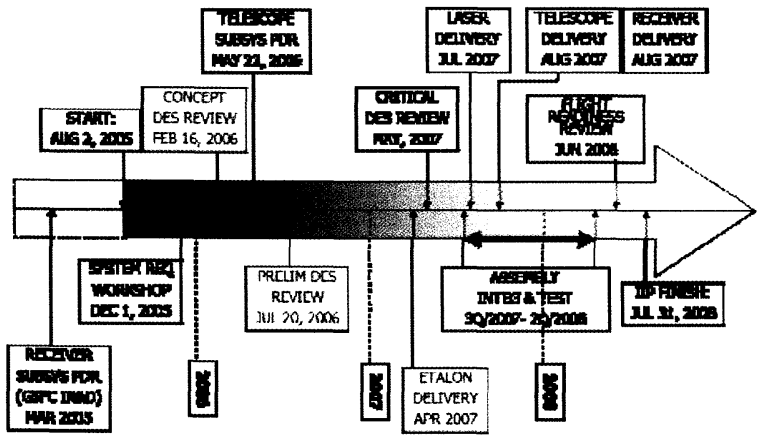

Figure 7 - TWiLiTE IIP Project timeline

challenge in the TWiLiTE design is integrating the instrument technologies into and end-to-end Doppler lidar system that functions autonomously in a relatively demanding environment. The WB57 payload bay is not environmentally controlled and temperatures on the ground may be greater than +40 degrees $\mathrm{C}$ while at altitude the temperature may be as low as -70 degrees $\mathrm{C}$ in the free air stream. Pressure at altitude will be 35 mbar. The TWiLiTE project is nearing the end of the second year of a scheduled three year effort. The design phase of the project is complete and major subsystem deliveries are scheduled to begin in July, 2007 with the delivery of the laser, followed by the HOE telescope and Dopler receiver in August. Integration of the TWiLiTE instrument and ground characterization and testing will be completed in 2008.

\section{Acknowledgement}

The TWiLITE project is funded by the NASA Earth Science Technology Office.

\section{References -}

Baker, W. et al, 1995: Lidar-Measured Winds from Space: A Key Component for Weather and Climate Prediction, Bulletin of the American Meteorological Society, 76, $869-888$.

Flesia, C. and C. Korb, (1999), "Theory of the doubleedge molecular technique for Doppler lidar wind measurement", Appl. Opt., 38, 432-440

Gentry, B., H. Chen and S. X. Li (2000). "Wind Measurements with a $355 \mathrm{~nm}$ Molecular Doppler Lidar", Optics Letters, 25, 1231-1233,

Gentry, B. and H. Chen (2002) "Performance validation and error analysis for a direct detection molecular Doppler lidar", SPIE Third International Asia-Pacific Environmental Remote Sensing Conference, Hangzhou, China, Oct 23-27, 2002.

Hovis, F., B. Gentry, M. Rhoades, R. Burnham, H. Chen and S. Li, "Single Frequency Lasers for Doppler Wind Lidar", 30th International Symposium on Remote Sensing of Environment, Honolulu, HI, Nov. 10-14, 2003.

McGill, M.J. ,W.D. Hart, J.A. McKay, and J.D. Spinhirne (1999), "Modeling the performance of directdetection Doppler lidar systems including cloud and solar background variability,", Appl. Opt., 38, 63886397.

Schwemmer, G., T. Wilkerson, and D. Guerra ,(1998) "Compact Scanning Lidar Systems Using Holographic Optics," SPIE Conf. Optical Remote Sensing for Industry and Environmental Monitoring, Beijing, China, 14-17 September 1998

Stoffelen, AA., Pailleux, J., Källén, E., Vaughan, J. M., Isaksen, L., Flamant, P., Wergen, W., Andersson, E., Schyberg, H., Culoma, A., Meynart, R., Endemann, M. and Ingmann, P. , 2005: The Atmospheric Dynamics Mission for Global Wind Field Measurement, Bulletin of the American Meteorological Society, 86, pp 73 - 87, 